\title{
Figures, Tables, and Map
}

\section{Figures}

1.1 Limited Nuclear War Thresholds

10.1 The Appropriateness of US Nuclear Responses to Adversary Actions

10.2 Geographic Areas Covered by Blast Effects at Varying Hardness

Tables

10.1 US Nuclear Forces, 2013 Vs. New START 213

10.2 Characteristics of US Strategic Forces 214

10.3 Matching US Nuclear Forces to Response Options 224

10.4 US ICBM Capability to Execute Limited Nuclear Attack Options Today

10.5 US SLBM Capability to Execute Limited Nuclear Attack Options Today 238

10.6 US Bomber Capability to Execute Limited Nuclear Attack Options Today

Map

10.1 Potential SSBN Patrol Areas to Minimize Overflight Concerns 
